\title{
Association of clinical characteristics, antidiabetic and cardiovascular agents with diabetes mellitus and COVID-19: a 7-month follow-up cohort study
}

\author{
Marzieh Pazoki ${ }^{1} \cdot$ Fatemeh Chichagi $^{2} \cdot$ Azar Hadadi $^{3} \cdot$ Samira Kafan $^{1} \cdot$ Mahnaz Montazeri $^{3} \cdot$ Sina Kazemian $^{4,5}$. \\ Arya Aminorroaya ${ }^{6} \cdot$ Mehdi Ebrahimi $^{7} \cdot$ Haleh Ashraf $^{2,5}$. Mojgan Mirabdolhagh Hazaveh ${ }^{7}$. \\ Mohammad Reza Khajavi $^{8} \cdot$ Reza Shariat Moharari $^{8} \cdot$ Seyed Hamidreza Sharifnia ${ }^{8}$. Shahrokh Karbalai Saleh ${ }^{9}$. \\ Hormat Rahimzadeh ${ }^{7} \cdot$ Neda Goodarzi $^{2} \cdot$ Peimaneh Heydarian ${ }^{7,10}$
}

Received: 12 July 2021 / Accepted: 13 September 2021 / Published online: 8 November 2021

(c) Springer Nature Switzerland AG 2021

\begin{abstract}
Background The prognostic factors of long-term outcomes in hospitalized patients with diabetes mellitus and COVID-19 are lacking.

Methods In this retrospective cohort study, we evaluated patients aged $\geq 18$-years-old with the COVID-19 diagnosis who were hospitalized between Feb 20 and Oct 29, 2020, in the Sina Hospital, Tehran, Iran. 1323 patients with COVID-19 entered in the final analysis, of whom 393 (29.7\%) patients had diabetes. We followed up patients for incurring in-hospital death, severe COVID-19, in-hospital complications, and 7-month all-cause mortality. By doing univariate analysis, variables with unadjusted P-value $<0.1$ in univariate analyses were regarded as the confounders to include in the logistic regression models. We made adjustments for possible clinical (model 1) and both clinical and laboratory (model 2) confounders.

Results After multivariable regression, it was revealed that preadmission use of sulfonylureas was associated with a borderline increased risk of severity in both models [model 1, OR (95\% CI):1.83 (0.91-3.71), P-value: 0.092; model 2, 2.05 (0.87-4.79), P-value: 0.099] and major adverse events (MAE: each of the severe COVID-19, multi-organ damage, or inhospital mortality) in model 1 [OR (95\% CI): 1.86 (0.90-3.87), P-value: 0.094]. Preadmission use of ACEIs/ARBs was associated with borderline increased risk of MAE in the only model 1 [OR (95\% CI):1.83 (0.96-3.48), P-value: 0.066]. Conclusions Preadmission use of sulfonylureas and ACEIs/ARBs were associated with borderline increased risk of inhospital adverse outcomes.
\end{abstract}

Keywords Diabetes mellitus $\cdot$ COVID-19 $\cdot$ SARS-CoV-2 $\cdot$ Antidiabetic agents $\cdot$ Long-term mortality

Marzieh Pazoki and Fatemeh Chichagi contributed equally to this work and shared co-first authorship.

Peimaneh Heydarian

heydarianpeimaneh@gmail.com

1 Department of Pulmonary Medicine, Sina Hospital, Tehran University of Medical Sciences, Tehran, Iran

2 Research Development Center, Sina Hospital, Tehran University of Medical Sciences, Tehran, Iran

3 Department of Infectious Diseases, Sina Hospital, Tehran University of Medical Sciences, Tehran, Iran

4 Students' Scientific Research Center (SSRC), Tehran University of Medical Sciences, Tehran, Iran

5 Cardiac Primary Prevention Research Center (CPPRC), Tehran Heart Center, Tehran University of Medical Sciences, Tehran, Iran
6 Non-Communicable Diseases Research Center, Endocrinology and Metabolism Population Sciences Institute, Tehran University of Medical Sciences, Tehran, Iran

7 Department of Internal Medicine, Sina Hospital, Tehran University of Medical Sciences, Tehran, Iran

8 Department of Anesthesiology, Sina Hospital, Tehran University of Medical Sciences, Tehran, Iran

9 Department of Cardiology, Sina Hospital, Tehran University of Medical Sciences, Tehran, Iran

10 Department of Endocrinology and Metabolism, Sina Hospital, Tehran University of Medical Sciences, Hasan Abad Sq., Tehran 11367-46911, Iran 


\section{Introduction}

In December 2019, new cases of atypical pneumonia were reported in Wuhan, China [1]. After detecting coronavirus disease-2019 (COVID-19), which is caused by the severe acute respiratory syndrome coronavirus-2 (SARS-CoV-2), the World Health Organization (WHO) announced the pandemic on March 11th, 2020 [2]. It has been reported patients with diabetes mellitus (DM), compared to those without $\mathrm{DM}$, are more likely to have a higher prevalence of hospitalization, severity and mortality of COVID-19, and DM has emerged as an important prognostic factor for severity and mortality in COVID-19 infection [3, 4].

According to previous studies, multiple factors including age, sex, ethnicity, and comorbidities, e.g.; obesity, hypertension, cardiovascular disease, and a pro-inflammatory and pro-coagulative state, probably contribute to the risk of worse outcomes in patients with DM and COVID-19 and beside these factors, some drugs which are commonly used by patients with DM like antidiabetic agents (ADAs) may play an important role [3, 4].

Observational studies suggested that among all the noninsulin antidiabetic agents, preadmission users of metformin had improved outcomes compared to the non-users [5-7]. The current evidence on the association of dipeptidyl-peptidase-4 (DPP-4) inhibitors and outcomes in patients with DM and COVID-19 is controversial $[8,9]$. Also, no documented harm or benefit has been reported for preadmission use of sulfonylureas in patients with DM and COVID-19 [10-12].

Angiotensin-converting enzyme inhibitors (ACEIs), angiotensin II receptor blockers (ARBs), and statins are commonly used medications by patients with DM; however, there are some uncertainties about using these drugs in patients with DM and COVID-19 [13]. Moreover, antiplatelets' beneficial, neutral, or hazardous effects are controversial [14-16].

In this retrospective cohort study, we aim to investigate whether there is an association between history of taking medications including ADAs (metformin, sulfonylureas, DPP-4 inhibitors, and insulin), and cardiovascular agents (ACEIs, ARBs, beta-blockers, calcium channel blockers, statins, and antiplatelets), with in-hospital outcomes and 7-month allcause mortality in hospitalized patients diagnosed with DM and COVID-19, in comparison with non-users.

\section{Methods}

\section{Ethical consideration}

The protocol of this study complied with the 2013 Helsinki declaration and was approved by the Research Ethics Committee of Tehran University of Medical Sciences
(IR.TUMS.VCR.REC.1399.018). We obtained written informed consent from all of the participants before inclusion in the study.

\section{Study design and population}

We evaluated electronic reports of 1413 patients admitted to Sina hospital, one of the major teaching hospitals for treating patients with COVID-19 in Tehran, Iran, between February 20 and October 29, 2020 as were partially included and defined in terms of treatment our primary reports [17-19]. The inclusion criteria for study participants were defined as patients with age $\geq 18$-year-old who had been hospitalized in Sina hospital with the diagnosis of COVID-19 according to upcoming criteria. Patients with a positive polymerase chain reaction (PCR) test for SARS-CoV-2 from oropharyngeal or endotracheal swab specimens and patients with ground-glass opacity, isolated or with consolidation in chest computed tomography (CT) scan which could not be considered as a result of volume overload, lobar or lung collapse, or nodules along with a compatible history of COVID-19 were diagnosed of having COVID-19 according to the World Health Organization's interim guidance [20] and the guideline of Iranian National Committee of COVID-19 [21]. We excluded 90 patients due to a lack of key information in their medical records. Finally, 1323 patients entered the final analysis. All patients were followed up using phone calls monthly after discharge. To ensure death information accuracy, we also checked the patients' national ID numbers in "National Organization for Civil Registration" on February 20, 2021; which were in line with hospital data for all patients. The median follow-up duration was 227.6 days with an interquartile range of 136.4 to 337.5 days, and the minimum follow-up duration was 117 days.

We retrieved the required data, including age, sex, body mass index (BMI), comorbidities, preadmission drug use, vital signs and laboratory data on admission, imaging findings, and treatments from electronic medical records which were completed by trained medical staff during the study period. Patients were treated by the latest guideline of the Iranian National Committee of COVID19 [22]; hence, these were subject to change during the study period due to rapidly updating literature. To control blood glucose levels during hospitalization non-insulin ADAs were discontinued and insulin infusion protocols, sliding scale, insulin intensification, or basal-bolus insulin regimen were considered for patients with diabetes. Patients were visited daily and followed up for in-hospital adverse outcomes including severity, acute respiratory distress syndrome (ARDS), acute cardiac injury (ACI), 
acute kidney injury (AKI), acute liver injury (ALI), and multi-organ damage.

\section{Definitions}

DM was defined as the history of taking ADA or according to the American Diabetes Association guidelines [23]. The systemic immune-inflammation index (SII) was calculated as (platelet count $\times$ neutrophil count)/(lymphocyte count). ACI was defined as an increased serum level of high-sensitivity cardiac troponin I (hs-cTnI) above the 99th percentile upper reference limit. The 99th percentile of hs-cTnI of our institution's laboratory kit was $11 \mathrm{pg} / \mathrm{mL}$ for women and $26 \mathrm{pg} / \mathrm{mL}$ for men [24]. ARDS was characterized based on the Berlin definition criteria [25]. AKI was defined as $\geq 0.3 \mathrm{mg} / \mathrm{dL}$ increase in serum creatinine within $48 \mathrm{~h}$, or $\geq 1.5$ times increase in baseline serum creatinine within

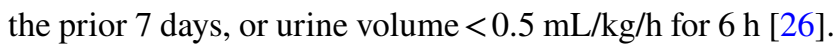
Patients with end-stage renal disease who required renal replacement therapy were excluded from this definition for AKI. ALI was defined as serum transaminases $\geq 3 \times$ upper limit normal (ULN) or alkaline phosphatase $\geq 2 \times$ ULN or total bilirubin $\geq 2 \times \mathrm{ULN}$ [27]. We defined multi-organ damage as the occurrence of at least two in-hospital complications, including ARDS, ACI, AKI, or ALI. Severe COVID-19 was characterized by the presence of each one of the following criteria: dyspnea, respiratory rate $\geq 30 / \mathrm{min}$, oxygen saturation $\leq 93 \%,>50 \%$ lung involvement on imaging, shock, respiratory failure, or multi-organ damage. We defined major adverse events (MAE) as the occurrence of each one of the severe COVID-19, multi-organ damage, or in-hospital mortality.

\section{Statistical analysis}

We indicated categorical variables by number (\%) and compared them using the Chi-square test. We examined the distribution of numerical variables using the Kolmogorov-Smirnov test and reported normally distributed ones as mean \pm standard deviation and non-normally distributed ones as median [interquartile range boundaries]. Independent samples t-test and the Mann-Whitney U test were employed to compare normally distributed and skewed variables. To investigate the possible effects of antidiabetic and cardiovascular agents on the outcomes (severe COVID-19, multi-organ damage, or 7-month all-cause mortality), we fitted binary logistic regression models. We did univariate analyses to indicate possible confounders of the study endpoints. Also, unadjusted odds ratio (OR), age-adjusted OR, and age-adjusted P-value were reported. The variables with unadjusted $\mathrm{P}$-value $<0.1$ in univariate analyses were considered confounders of the correspondent endpoint and included in the logistic regression models. We employed multivariate cox regression models for evaluating the effects of the medications on severity, MAE, and 7-month all-cause mortality. In Model 1 , multivariate regression was adjusted for age, sex, and comorbidities (hypertension, cardiac disease, cerebrovascular disease, chronic lung disease, malignancy), and in Model 2, multivariate regression was adjusted for age, sex, comorbidities (hypertension, cardiac disease, cerebrovascular disease, chronic lung disease, malignancy), white blood cells (WBC), C-reactive protein (CRP), urea, and aspartate aminotransferase (AST). The Hosmer and Lemeshow test was performed to evaluate the goodness of fit for logistic regression models. All data were analyzed using IBM Corp. Released in 2016. IBM SPSS Statistics for Windows (Version 24.0. Armonk, NY: IBM Corp).

\section{Results}

\section{Demographics and baseline characteristics in patients with DM and COVID-19}

In this study, we included 1323 patients with COVID19, of whom 393 (29.7\%) patients had diabetes. In the whole cohort, the mean age was $59.2 \pm 16.5$ years, and $808(61.1 \%)$ were males. The most common comorbidities were hypertension (45.5\%), diabetes $(29.7 \%)$, and cardiac disease (23.3\%). Patients with diabetes compared to those without diabetes were significantly older, more male, and had higher BMI. The prevalence of underlying diseases such as hypertension, cardiac disease, cerebrovascular disease, and chronic kidney disease was significantly higher in patients with diabetes compared to those without diabetes. Patients with diabetes had significantly longer hospital length of stay (P-value $<0.001$ ), increased risk of severe COVID-19 (P-value $<0.001$ ), need for mechanical ventilation (P-value: 0.013), multi-organ damage (P-value $<0.001$ ), and in-hospital, 1-month and 7-month all-cause mortality (Supplementary Table 1). The percentage of in-hospital, 1-month and 7-month allcause deaths in patients with diabetes compared to those without diabetes were significantly higher; 23.4 vs. $15.4 \%$ (P-value <0.001), 24.4 vs. 18.0\% (P-value: 0.007), and 30.5 vs. $21.4 \%$ (P-value $<0.001)$ respectively.

\section{Prognostic factors of severity, MAE, and 7-month all-cause mortality in patients with DM and COVID-19}

The severe COVID-19 and MAE developed in 304 (77.4\%) and $310(78.9 \%)$ patients, respectively. After adjustment for 
age, univariate analyses demonstrated that higher systolic blood pressure; fever; lower oxygen saturation; higher WBC, absolute neutrophil counts, neutrophil-to-lymphocyte ratio, SII, CRP, and AST were associated with increased severity (Supplementary Table 2), increased MAE (Table 1), and higher 7-month all-cause mortality (Table 2) (All P-values $<0.05$ ). Also, higher alanine aminotransferase (ALT) was associated with increased severity $(\mathrm{P}-$ value $<0.001)$ and higher MAE (P-value $<0.001)$. The lower level of sodium was only associated with severity (P-value: 0.027 ).

The male sex; the history of cerebrovascular disease and malignancy; higher diastolic blood pressure, urea, creatinine, blood urea nitrogen (BUN) to creatinine ratio, hs-c Tnl; and all adverse in-hospital outcomes were also associated with higher 7-month all-cause mortality (All P-values $<0.05$ ).

\section{Effect of preadmission use of antidiabetic and cardiovascular agents on clinical outcomes}

To investigate the independent effects of preadmission-use of anti-diabetic and cardiovascular agents on severity, MAE, and 7-month all-cause mortality; after adjustment for possible clinical (model 1) and clinical and laboratory (model 2) confounders, preadmission use of sulfonylureas was associated with a borderline increased risk of severity in both models [model 1, OR (95\% CI): 1.83 (0.91-3.71), P-value: 0.092; model 2, 2.05 (0.87-4.79), P-value: 0.099] and MAE in model 1 [OR (95\% CI): 1.86 (0.90-3.87), P-value: 0.094]. Preadmission use of ACEIs/ARBs was associated with borderline increased risk of MAE in only model 1 [OR (95\% CI): 1.83 (0.96-3.48), P-value: 0.066] (Table 3).

\section{Discussion}

In this retrospective cohort study, the percentage of in-hospital deaths, 1-month deaths, and 7-month all-cause deaths among 393 (29.7\%) patients with DM and COVID-19, compared to those without diabetes, were significantly higher. In a recent meta-analysis and meta-regression, sourcing data of 45,775 hospitalized COVID-19 patients, a weighted prevalence of mortality in hospitalized patients with DM and COVID-19 was 1.82-times higher than in COVID-19 patients without diabetes, and this proportion was even more among critically ill patients [28].

According to various reports, Older age [29], male sex [30], obesity [30], hypertension [31], pre-existing cardiocerebrovascular diseases [30], chronic kidney disease [32], acute kidney injury [33], pro-inflammatory [34], and procoagulative state [35] were associated with severity and/or short-term mortality in COVID-19 patients.

In this study, to find out the factors which contribute to long term mortality more precisely, we also did an age-adjusted univariate analysis, which identified several risk factors for 7-month all-cause mortality in patients with DM and COVID-19, including male sex, comorbidities such as cerebrovascular disease, malignancy, hypertension, and kidney dysfunction indices e.g. higher urea, creatinine, and blood urea nitrogen to creatinine ratio; pro-inflammatory indices such as fever, higher WBC, absolute neutrophil counts, neutrophil-to-lymphocyte ratio, SII, CRP and AST; and coagulative state e.g. higher hs-cardiac troponin I. Also, adverse in-hospital outcomes, e.g., hospital length of stay, severe COVID-19, ICU admission, and multi-organ damage, were predictors for 7-month all-cause mortality, compatible with previous reports.

In this study, preadmission use of sulfonylureas was associated with borderline increased risk of in-hospital adverse outcomes. In the pre-pandemic era, meta-regression analysis of the observational studies showed that sulfonylureas were associated with an increased risk of cardiovascular events and mortality in patients with DM [36]. Meta-analysis of randomized clinical trials suggested that the use of secondand third-generation sulfonylureas in patients with type 2 DM was not associated with increased cardiovascular risk and all-cause mortality, irrespective of comparator or background medication [37].

Patients who use sulfonylureas are at greater risk of hypoglycemia, especially in the hospital setting [38-40]; Nonetheless, no evidence has been reported beneficial or detrimental effects for preadmission use of sulfonylureas in patients with DM and COVID-19 yet [10-12].

The present study found other ADA had no impact on outcomes in patients with diabetes and COVID-19.

Observational studies suggested that among all the noninsulin ADA, preadmission users of metformin had improved outcomes compared to the non-users [5]. The interim report of the prospective CORONADO study showed no difference in both in-hospital and 7-day mortality regarding different ADA in patients with DM and COVID-19. However, only preadmission metformin users had a lower rate of death compared to the non-users in an unadjusted but not in the multivariate analysis [6]. Also, metformin users had a significant $60 \%$ increased hospital discharge rate and a $35 \%$ reduced 28 -day mortality rate than the non-users in the multivariate analysis [7]. A meta-analysis done on 10,233 patients with DM revealed metformin use is associated with a lower mortality rate in non-adjusted [OR(95\% CI): 0.45 (0.25-0.81), P-value: 0.008$]$ and adjusted [OR(95\% CI): 0.64 (0.43-0.97), P-value: 0.035] model [5]; nevertheless, a retrospective observational study on 6256 patients with diabetes and COVID-19 showed an association between metformin and reduced risk of mortality only in women [OR(95\% CI): 0.79 (0.64-0.98), P-value: 0.013] but not in men [40]. Another observational and multi-centric study, including 2666 patients with DM and COVID-19, showed 
Table 1 Characteristics associated with MAE in patients with DM and COVID-19

\begin{tabular}{|c|c|c|c|c|c|c|}
\hline \multirow[t]{2}{*}{ Characteristic* } & \multirow[t]{2}{*}{ Total $(\mathrm{N}=393)$} & \multicolumn{5}{|l|}{ MAE } \\
\hline & & $\operatorname{MAE}(\mathrm{N}=310)$ & Non-MAE (N=83) & $\begin{array}{l}\text { Unadjusted OR } \\
(95 \% \mathrm{CI})\end{array}$ & $\begin{array}{l}\text { Age-adjusted OR } \\
\text { (95\% CI) }\end{array}$ & Age-adjusted $\mathrm{P}^{\dagger}$ \\
\hline \multicolumn{7}{|l|}{ Demographics } \\
\hline Age (year) & $65.4 \pm 11.6$ & $66.07 \pm 11.60$ & $62.93 \pm 11.50$ & $1.02(1.00-1.05)$ & - & - \\
\hline \multicolumn{7}{|l|}{ Sex } \\
\hline Female & $172(43.8 \%)$ & $139(44.8 \%)$ & $33(39.8 \%)$ & $1.23(0.75-2.02)$ & $1.22(0.74-2.01)$ & 0.424 \\
\hline Male & $221(56.2 \%)$ & $171(55.2 \%)$ & $50(60.2 \%)$ & & & \\
\hline BMI $\left(\mathrm{kg} / \mathrm{m}^{2}\right)$ & $28.0 \pm 5.1$ & $28.15 \pm 5.23$ & $27.70 \pm 4.43$ & $1.02(0.96-1.08)$ & $1.02(0.96-1.09)$ & 0.453 \\
\hline \multicolumn{7}{|l|}{ Comorbidities } \\
\hline Hypertension & $257(65.4 \%)$ & $207(66.7 \%)$ & $50(60.2 \%)$ & $1.33(0.80-2.18)$ & $1.12(0.66-1.90)$ & 0.670 \\
\hline Cardiac disease & $131(33.3 \%)$ & $105(33.9 \%)$ & $26(31.3 \%)$ & $1.12(0.67-1.90)$ & $0.96(0.56-1.65)$ & 0.88 \\
\hline $\begin{array}{l}\text { Cerebrovascular } \\
\text { disease }\end{array}$ & $32(8.1 \%)$ & $30(9.7 \%)$ & $2(2.4 \%)$ & $4.34(1.01-18.55)$ & $3.79(0.88-16.36)$ & 0.074 \\
\hline $\begin{array}{l}\text { Chronic lung } \\
\text { disease }\end{array}$ & $22(5.6 \%)$ & $19(6.1 \%)$ & $3(3.6 \%)$ & $1.74(0.50-6.03)$ & $1.58(0.45-5.53)$ & 0.747 \\
\hline $\begin{array}{l}\text { Chronic kidney } \\
\text { disease }\end{array}$ & $31(7.9 \%)$ & $26(8.4 \%)$ & $5(6.0 \%)$ & $1.43(0.53-3.84)$ & $1.32(0.49-3.57)$ & 0.588 \\
\hline Malignancy & $22(5.6 \%)$ & $19(6.1 \%)$ & $3(3.6 \%)$ & $1.74(0.50-6.03)$ & $1.52(0.434-5.33)$ & 0.512 \\
\hline \multicolumn{7}{|c|}{ Preadmission drug use } \\
\hline Metformin & $177(45.0 \%)$ & $143(46.1 \%)$ & $34(41.0 \%)$ & $1.23(0.75-2.02)$ & $1.34(0.81-2.20)$ & 0.252 \\
\hline Sulfonylureas & $72(18.3 \%)$ & $62(20.0 \%)$ & $10(12.0 \%)$ & $1.82(0.89-3.74)$ & $1.80(0.88-3.71)$ & 0.109 \\
\hline $\begin{array}{l}\text { DPP-4 } \\
\text { inhibitors }\end{array}$ & $20(5.1 \%)$ & $16(5.2 \%)$ & $4(4.8 \%)$ & $1.07(0.35-3.31)$ & $1.24(0.40-3.88)$ & 0.707 \\
\hline Insulin & $53(13.5 \%)$ & $41(13.2 \%)$ & $12(14.5 \%)$ & $0.90(0.45-1.81)$ & $0.82(0.40-1.65)$ & 0.574 \\
\hline ACEIs/ARBs & $128(32.6 \%)$ & $109(35.2 \%)$ & $19(22.9 \%)$ & $1.83(1.04-3.21)$ & $1.69(0.95-2.99)$ & 0.072 \\
\hline Beta blockers & $75(19.1 \%)$ & $62(20.0 \%)$ & $13(15.7 \%)$ & $1.35(0.70-2.59)$ & $1.29(0.67-2.49)$ & 0.452 \\
\hline $\begin{array}{l}\text { Calcium chan- } \\
\text { nel blockers }\end{array}$ & $37(9.4 \%)$ & $33(10.6 \%)$ & $4(4.8 \%)$ & $2.35(0.81-6.84)$ & $2.16(0.74-6.33)$ & 0.159 \\
\hline Statins & $107(27.2 \%)$ & $91(29.4 \%)$ & $16(19.3 \%)$ & $1.74(0.96-3.16)$ & $1.68(0.92-3.06)$ & 0.092 \\
\hline Antiplatelets & $110(28.0 \%)$ & $94(30.3 \%)$ & $16(19.3 \%)$ & $1.82(1.00-3.31)$ & $1.66(0.91-3.06)$ & 0.100 \\
\hline \multicolumn{7}{|c|}{ Vital signs on admission } \\
\hline $\begin{array}{l}\text { Heart rate } \\
\quad \text { (beat } / \mathrm{min} \text { ) }\end{array}$ & $90.9 \pm 14.6$ & $91.61 \pm 15.26$ & $87.93 \pm 11.38$ & $1.02(1.00-1.04)$ & $1.02(1.00-1.04)$ & 0.035 \\
\hline $\begin{array}{l}\text { Systolic blood } \\
\text { pressure } \\
(\mathrm{mmHg})\end{array}$ & $129.0 \pm 20.9$ & $127.94 \pm 20.63$ & $132.77 \pm 21.77$ & $0.99(0.98-1.00)$ & $0.99(0.98-1.00)$ & 0.051 \\
\hline $\begin{array}{l}\text { Diastolic blood } \\
\text { pressure } \\
(\mathrm{mmHg})\end{array}$ & $76.8 \pm 13.0$ & $76.53 \pm 13.33$ & $77.97 \pm 11.92$ & $0.99(0.97-1.01)$ & $0.99(0.97-1.01)$ & 0.401 \\
\hline $\begin{array}{l}\text { Temperature } \\
\left({ }^{\circ} \mathrm{C}\right)\end{array}$ & $37.2 \pm 0.9$ & $37.23 \pm 0.93$ & $36.92 \pm 0.63$ & $1.57(1.12-2.20)$ & $1.60(1.13-2.25)$ & 0.007 \\
\hline $\begin{array}{l}\text { Oxygen satura- } \\
\text { tion }(\%)\end{array}$ & $88.4 \pm 7.9$ & $87.09 \pm 8.05$ & $94.63 \pm 2.72$ & $0.68(0.61-0.76)$ & $0.68(0.61-0.76)$ & 0.000 \\
\hline \multicolumn{7}{|c|}{ Laboratory data on admission } \\
\hline $\mathrm{WBC}\left(\times 10^{9} / \mathrm{L}\right)$ & $7.0[5.2-9.6]$ & $7.5[5.3-9.8]$ & $6.0[4.8-8.5]$ & $1.10(1.02-1.19)$ & $1.09(1.01-1.18)$ & 0.036 \\
\hline $\begin{array}{l}\text { Neutrophil } \\
\left(\times 10^{9} / \mathrm{L}\right)\end{array}$ & $1.1[0.8-1.6]$ & $5.7[3.7-8.0]$ & $4.3[3.0-6.2]$ & $1.14(1.04-1.25)$ & $1.13(1.03-1.04)$ & 0.011 \\
\hline $\begin{array}{l}\text { Lymphocyte } \\
\left(\times 10^{9} / \mathrm{L}\right)\end{array}$ & $1.1[0.8-1.6]$ & $1.1[0.8-1.5]$ & $1.4[1.0-1.8]$ & $0.88(0.69-1.13)$ & $0.86(0.67-1.10)$ & 0.229 \\
\hline $\begin{array}{l}\text { Platelets } \\
\left(\times 10^{9} / \mathrm{L}\right)\end{array}$ & $\begin{array}{l}189.0 \\
{[147.7-263.0]}\end{array}$ & $191.0[149-265]$ & $187.0[145-248]$ & $1.00(1.00-1.00)$ & $1.00(1.00-1.00)$ & 0.134 \\
\hline
\end{tabular}


Table 1 (continued)

\begin{tabular}{|c|c|c|c|c|c|c|}
\hline \multirow[t]{2}{*}{ Characteristic* } & \multirow[t]{2}{*}{ Total $(\mathrm{N}=393)$} & \multicolumn{5}{|l|}{ MAE } \\
\hline & & $\operatorname{MAE}(\mathrm{N}=310)$ & Non-MAE (N=83) & $\begin{array}{l}\text { Unadjusted OR } \\
(95 \% \mathrm{CI})\end{array}$ & $\begin{array}{l}\text { Age-adjusted OR } \\
(95 \% \mathrm{CI})\end{array}$ & Age-adjusted $\mathrm{P}^{\dagger}$ \\
\hline $\begin{array}{l}\text { Neutrophil-to- } \\
\text { lymphocyte } \\
\text { ratio }\end{array}$ & $4.4[2.7-7.8]$ & $4.7[3.0-8.4]$ & $3.3[2.0-5.2]$ & $1.16(1.06-1.27)$ & $1.16(1.6-1.27)$ & 0.001 \\
\hline SII & $\begin{array}{l}844.4 \\
\quad[468.2-1695.2]\end{array}$ & $\begin{array}{l}928.9 \\
{[1790.5-486.0]}\end{array}$ & $\begin{array}{l}592.7 \\
{[369.0-1076.3]}\end{array}$ & $1.00(1.00-1.00)$ & $1.00(1.00-1.00)$ & 0.001 \\
\hline $\mathrm{RBC}\left(\times 10^{12} / \mathrm{L}\right)$ & $4.5[4.0-5.0]$ & $4.6[4.0-5.0]$ & $4.5[4.1-5.0]$ & $1.08(0.76-1.53)$ & $1.17(0.82-1.67)$ & 0.387 \\
\hline $\begin{array}{l}\text { Hemoglobin } \\
(\mathrm{g} / \mathrm{dL})\end{array}$ & 13.0]11.5-14.6] & $13.0[11.5-14.7]$ & $13.0[11.6-14.3]$ & $1.00(0.89-1.12)$ & $1.01(0.90-1.14)$ & 0.805 \\
\hline Urea (mg/dL) & $40.0[28.0-65.0]$ & $41.0[29.0-68.0]$ & $34.0[25.0-49.0]$ & $1.01(1.00-1.02)$ & $1.01(1.00-1.02)$ & 0.084 \\
\hline $\begin{array}{l}\text { Creatinine } \\
(\mathrm{mg} / \mathrm{dL})\end{array}$ & $1.1[0.9-1.5]$ & $1.2[0.9-1.6]$ & $1.0[0.9-1.4]$ & $1.19(0.88-1.62)$ & $1.15(0.86-1.53)$ & 0.353 \\
\hline BUN/creatinine & $15.9[12.1-20.5]$ & $16.0[12.4-20.6]$ & $14.8[11.4-20.1]$ & $1.05(1.00-1.09)$ & $1.04(0.99-1.08)$ & 0.092 \\
\hline $\begin{array}{l}\text { Sodium } \\
(\mathrm{mmol} / \mathrm{L})\end{array}$ & $\begin{array}{l}135.1 \\
\quad[131.6-138.6]\end{array}$ & $\begin{array}{l}134.8 \\
{[131.3-138.5]}\end{array}$ & $\begin{array}{l}136.3 \\
{[132.2-138.9]}\end{array}$ & $0.97(0.93-1.01)$ & $0.97(0.93-1.01)$ & 0.190 \\
\hline $\begin{array}{l}\text { Potassium } \\
(\mathrm{mmol} / \mathrm{L})\end{array}$ & $4.5[4.1-4.8]$ & $4.5[4.1-4.9]$ & $4.4[4.1-4.7]$ & $1.15(0.74-1.79)$ & $1.10(0.70-1.72)$ & 0.687 \\
\hline $\begin{array}{l}\text { Blood sugar } \\
(\mathrm{mg} / \mathrm{dL})\end{array}$ & $\begin{array}{l}172.0 \\
{[118.0-244.0]}\end{array}$ & $181.0[120-258]$ & $\begin{array}{l}161.0 \\
{[105.0-229.0]}\end{array}$ & $1.00(1.00-1.00)$ & $1.00(1.00-1.01)$ & 0.144 \\
\hline FBS (mg/dL) & $\begin{array}{l}193.0 \\
{[141.5-259.0]}\end{array}$ & $\begin{array}{l}192.0 \\
{[140.0-252.7]}\end{array}$ & $\begin{array}{l}219.0 \\
{[155.5-277.5]}\end{array}$ & $1.00(1.00-1.00)$ & $1.00(1.00-1.00)$ & 0.782 \\
\hline CRP (mg/L) & 68.1 [31.2-103.6] & $73.2[38.0-118.3]$ & $48.5[11.7-78.5]$ & $1.01(1.00-1.02)$ & $1.01(1.00-1.02)$ & 0.001 \\
\hline $\mathrm{ESR}(\mathrm{mm} / \mathrm{h})$ & 61.5 [37.0-90.0] & $64.0[39.0-90.0]$ & $54.0[30.0-84.7]$ & $1.01(1.00-1.02)$ & $1.01(1.00-1.02)$ & 0.077 \\
\hline $\begin{array}{l}\text { hs-cTnI } \\
\quad(p g / m L)\end{array}$ & $6.0[1.8-16.1]$ & $7.1[2.4-25.2]$ & $2.6[1.5-9.4]$ & $1.00(1.00-1.01)$ & $1.00(1.00-1.01)$ & 0.326 \\
\hline AST (U/L) & $52.0[37.0-68.0]$ & $55.0[41.0-72.5]$ & $36.0[28.0-50.0]$ & $1.05(1.03-1.07)$ & $1.05(1.03-1.08)$ & 0.000 \\
\hline ALT (U/L) & $37.0[27.0-52.0]$ & $40.0[29.0-55.0]$ & $29.5[22.0-37.7]$ & $1.05(1.02-1.07)$ & $1.05(1.02-1.07)$ & 0.000 \\
\hline ALP (U/L) & $\begin{array}{l}176.5 \\
{[140.2-231.0]}\end{array}$ & $\begin{array}{l}176.0 \\
{[140.7-233.2]}\end{array}$ & $\begin{array}{l}187.0 \\
{[138.7-222.5]}\end{array}$ & $1.00(1.00-1.00)$ & $1.00(1.00-1.00)$ & 0.307 \\
\hline \multicolumn{7}{|c|}{ In-hospital outcomes } \\
\hline $\begin{array}{l}\text { Hospital length } \\
\text { of stay (day) }\end{array}$ & $6.0[3.0-9.5]$ & $6.0[4.0-10.0]$ & $4.0[2.0-7.0]$ & $1.06(1.01-1.11)$ & $1.06(1.01-1.11)$ & 0.028 \\
\hline
\end{tabular}

ACEIs angiotensin-converting-enzyme inhibitors, $A L P$ alkaline phosphatase, $A L T$ alanine aminotransferase, $A S T$ aspartate aminotransferase, $A R B s$ angiotensin II receptor blockers, $B M I$ body mass index, $B U N$ blood urea nitrogen, $C R P$ C-reactive protein, DPP-4 inhibitors dipeptidyl peptidase-4 inhibitors, $E S R$ erythrocyte sedimentation rate, $F B S$ fasting blood sugar, $h s$-cTnI high-sensitivity cardiac troponin I, $M A E$ major adverse event, $R B C$ red blood cells, SII systemic immune-inflammation index, $W B C$ white blood cells

*Data are presented as mean \pm standard deviation, number (\%), or median [interquartile range]

${ }^{\dagger}$ Statistically significant P-values are bolded

that metformin had neutral effects on death and adverse outcomes compatible with our findings [41]. On the contrary, a case-control study done on 110 patients with diabetes and COVID-19 showed metformin users in comparison with non-users, are more likely to experience life-threatening complications including ARDS, septic shock, sepsis, organ dysfunctions leading to ICU admission (28.6\% vs. $7.4 \%$, P-value: 0.004$)$ and progressive disease [adjusted OR(95\% CI): 3.96 (1.03-15.19), P-value: 0.045] [42].

The current evidence on the association of dipeptidylpeptidase-4 (DPP-4) inhibitors and outcomes in patients with DM and COVID-19 is controversial [8, 9].
The insulin used by patients with diabetes and COVID-19 is a contentious subject. Several studies showed preadmission insulin use is associated with a higher risk of within 28 days [OR (95\% CI): 1.34 (0.95-1.91), P-value: 0.010] and in-hospital [OR(95\% CI): 2.30 (1.32-4.01)] death [43]. By contrast, an observational study including 239 patients with DM and COVID-19 found no association between preadmission insulin use and the risk of mortality [44].

Our study showed that ACEIs/ARBs were associated with borderline increased risk of severe COVID-19, multiorgan damage, or in-hospital mortality. Despite the fact that there are some uncertainties about taking these drugs 
Table 2 Characteristics associated with 7-month all-cause mortality in patients with DM and COVID-19

\begin{tabular}{|c|c|c|c|c|c|c|}
\hline \multirow[t]{2}{*}{ Characteristic* } & \multirow[t]{2}{*}{ Total $(\mathrm{N}=393)$} & \multicolumn{5}{|c|}{ 7-month all-cause mortality } \\
\hline & & Deceased $(\mathrm{N}=120)$ & Survived $(\mathrm{N}=273)$ & $\begin{array}{l}\text { Unadjusted OR } \\
(95 \% \mathrm{CI})\end{array}$ & $\begin{array}{l}\text { Age-adjusted OR } \\
(95 \% \text { CI })\end{array}$ & Age-adjusted $\mathrm{P} \dagger$ \\
\hline \multicolumn{7}{|l|}{ Demographics } \\
\hline Age (year) & $65.4 \pm 11.6$ & $70.2 \pm 11.7$ & $63.3 \pm 11.0$ & $1.06(1.03-1.08)$ & - & - \\
\hline \multicolumn{7}{|l|}{ Sex } \\
\hline Female & $172(43.8 \%)$ & $44(36.7 \%)$ & $128(46.9 \%)$ & $0.66(0.42-1.02)$ & $0.61(0.38-0.96)$ & 0.035 \\
\hline Male & $221(56.2 \%)$ & $76(63.3 \%)$ & $145(53.1 \%)$ & & & \\
\hline BMI $\left(\mathrm{kg} / \mathrm{m}^{2}\right)$ & $28.0 \pm 5.1$ & $26.7 \pm 4.6$ & $28.5 \pm 5.1$ & $0.93(0.87-0.99)$ & $0.94(0.88-1.00)$ & 0.071 \\
\hline \multicolumn{7}{|l|}{ Comorbidities } \\
\hline Hypertension & $257(65.4 \%)$ & $81(67.5 \%)$ & $176(64.5 \%)$ & $1.14(0.73-1.80)$ & $0.72(0.44-1.19)$ & 0.205 \\
\hline Cardiac disease & $131(33.3 \%)$ & $44(36.7 \%)$ & $87(31.9 \%)$ & $1.24(0.79-1.94)$ & $0.85(0.52-1.39)$ & 0.527 \\
\hline $\begin{array}{l}\text { Cerebrovascular } \\
\text { disease }\end{array}$ & $32(8.1 \%)$ & $17(14.2 \%)$ & $15(5.5 \%)$ & $2.84(1.37-5.90)$ & $2.14(1.00-4.59)$ & 0.050 \\
\hline $\begin{array}{l}\text { Chronic lung } \\
\text { disease }\end{array}$ & $22(5.6 \%)$ & $10(8.3 \%)$ & $12(4.4 \%)$ & $1.98(0.83-4.71)$ & $1.71(0.68-4.30)$ & 0.257 \\
\hline $\begin{array}{l}\text { Chronic kidney } \\
\text { disease }\end{array}$ & $31(7.9 \%)$ & $11(9.2 \%)$ & $20(7.3 \%)$ & $1.28(0.59-2.75)$ & $1.07(0.48-2.39)$ & 0.876 \\
\hline Malignancy & $22(5.6 \%)$ & $14(11.7 \%)$ & $8(2.9 \%)$ & $4.37(1.78-10.73)$ & $3.57(1.40-9.09)$ & 0.008 \\
\hline \multicolumn{7}{|c|}{ Preadmission drug use } \\
\hline Metformin & $177(45.0 \%)$ & $48(40.0 \%)$ & $129(47.3 \%)$ & $0.74(0.48-1.15)$ & $0.87(0.55-1.38)$ & 0.566 \\
\hline Sulfonylureas & $72(18.3 \%)$ & $23(19.2 \%)$ & $49(17.9 \%)$ & $1.08(.63-1.88)$ & $1.04(0.59-1.85)$ & 0.890 \\
\hline $\begin{array}{l}\text { DPP-4 } \\
\text { inhibitors }\end{array}$ & $20(5.1 \%)$ & $5(4.2 \%)$ & $15(5.5 \%)$ & $0.75(0.26-2.11$ & $1.02(0.35-3.01)$ & 0.967 \\
\hline Insulin & $53(13.5 \%)$ & $16(13.3 \%)$ & $37(13.6 \%)$ & $0.98(0.52-1.84)$ & $0.79(0.41-1.53)$ & 0.493 \\
\hline ACEIs/ARBs & $128(32.6 \%)$ & $40(33.3 \%)$ & $88(32.2 \%)$ & $1.05(0.67-1.66)$ & $0.84(0.52-1.36)$ & 0.482 \\
\hline Beta blockers & $75(19.1 \%)$ & $21(17.5 \%)$ & $54(19.8 \%)$ & $0.86(0.49-1.50)$ & $0.78(0.43-1.38)$ & 0.391 \\
\hline $\begin{array}{c}\text { Calcium chan- } \\
\text { nel blockers }\end{array}$ & $37(9.4 \%)$ & $13(10.8 \%)$ & $24(8.8 \%)$ & $1.26(0.62-2.57)$ & $1.05(0.50-2.23)$ & 0.893 \\
\hline Statins & $107(27.2 \%)$ & $26(21.7 \%)$ & $81(29.7 \%)$ & $0.66(0.39-1.09)$ & $0.59(0.35-1.01)$ & 0.053 \\
\hline Antiplatelets & $110(28.0 \%)$ & $34(28.3 \%)$ & $76(27.8 \%)$ & $1.02(0.64-1.65)$ & $0.81(0.49-1.34)$ & 0.412 \\
\hline \multicolumn{7}{|c|}{ Vital signs on admission } \\
\hline $\begin{array}{l}\text { Heart rate } \\
\text { (beat/min) }\end{array}$ & $90.9 \pm 14.6$ & $92.4 \pm 16.6$ & $90.2 \pm 13.6$ & $1.01(0.99-1.03)$ & $1.01(1.0-1.03)$ & 0.078 \\
\hline $\begin{array}{l}\text { Systolic blood } \\
\text { pressure } \\
(\mathrm{mmHg})\end{array}$ & $129.0 \pm 20.9$ & $126.0 \pm 20.7$ & $130.4 \pm 21.0$ & $0.99(0.98-1.00)$ & $0.98(0.97-1.00)$ & 0.013 \\
\hline $\begin{array}{l}\text { Diastolic blood } \\
\text { pressure } \\
(\mathrm{mmHg})\end{array}$ & $76.8 \pm 13.0$ & $74.3 \pm 12.6$ & $78.0 \pm 13.1$ & $0.98(0.96-1.00)$ & $0.97(0.95-0.99)$ & 0.011 \\
\hline Temperature $\left({ }^{\circ} \mathrm{C}\right)$ & $37.2 \pm 0.9$ & $37.4 \pm 1.0$ & $37.1 \pm 0.8$ & $1.48(1.15-1.92)$ & $1.56(1.19-2.04)$ & 0.001 \\
\hline $\begin{array}{l}\text { Oxygen satura- } \\
\text { tion }(\%)\end{array}$ & $88.4 \pm 7.9$ & $85.0 \pm 10.6$ & $89.9 \pm 6.0$ & $0.93(0.90-0.96)$ & $0.92(0.89-0.95)$ & $<0.001$ \\
\hline \multicolumn{7}{|c|}{ Laboratory data on admission } \\
\hline WBC $\left(\times 10^{9} / \mathrm{L}\right)$ & $7.0[5.2-9.6]$ & $8.6[5.6-12.0]$ & $6.7[5.1-8.7]$ & $1.15(1.08-1.22)$ & $1.13(1.06-1.20)$ & $<0.001$ \\
\hline $\begin{array}{l}\text { Neutrophil } \\
\left(\times 10^{9} / \mathrm{L}\right)\end{array}$ & $1.1[0.8-1.6]$ & $6.8[4.2-9.2]$ & $4.9[3.4-7.1]$ & $1.16(1.08-1.24)$ & $1.14(1.06-1.22)$ & $<0.001$ \\
\hline $\begin{array}{l}\text { Lymphocyte } \\
\left(\times 10^{9} / \mathrm{L}\right)\end{array}$ & $1.1[0.8-1.6]$ & $1.1[0.8-1.5]$ & $1.2[0.9-1.6]$ & $1.11(0.88-1.40)$ & $1.06(0.83-1.35)$ & 0.629 \\
\hline $\begin{array}{l}\text { Platelets } \\
\left(\times 10^{9} / \mathrm{L}\right)\end{array}$ & $\begin{array}{l}189.0 \\
{[147.7-263.0]}\end{array}$ & $\begin{array}{l}189.0 \\
{[144.0-253.7]}\end{array}$ & $\begin{array}{l}190.0 \\
{[148.7-267.2]}\end{array}$ & 1.00 & 1.00 & 0.431 \\
\hline $\begin{array}{l}\text { Neutrophil-to- } \\
\text { lymphocyte } \\
\text { ratio }\end{array}$ & $4.4[2.7-7.8]$ & $5.9[3.6-9.8]$ & $3.9[2.5-6.7]$ & $1.09(1.03-1.15)$ & $1.08(1.03-1.14)$ & 0.003 \\
\hline
\end{tabular}


Table 2 (continued)

\begin{tabular}{|c|c|c|c|c|c|c|}
\hline \multirow[t]{2}{*}{ Characteristic* } & \multirow[t]{2}{*}{ Total $(\mathrm{N}=393)$} & \multicolumn{5}{|c|}{ 7-month all-cause mortality } \\
\hline & & Deceased $(\mathrm{N}=120)$ & Survived $(N=273)$ & $\begin{array}{l}\text { Unadjusted OR } \\
(95 \% \mathrm{CI})\end{array}$ & $\begin{array}{l}\text { Age-adjusted OR } \\
(95 \% \mathrm{CI})\end{array}$ & Age-adjusted $\mathrm{P} \dagger$ \\
\hline SII & $\begin{array}{l}844.4 \\
\quad[468.2-1695.2]\end{array}$ & $\begin{array}{l}1018.2 \\
{[543.2-2216.1]}\end{array}$ & $\begin{array}{l}786.9 \\
{[448.2-1514.1]}\end{array}$ & 1.00 & 1.00 & 0.022 \\
\hline $\mathrm{RBC}\left(\times 10^{12} / \mathrm{L}\right)$ & $4.5[4.0-5.0]$ & $4.4[3.9-5.0]$ & $4.6[4.1-5.1]$ & $0.76(0.56-1.02)$ & $0.87(0.63-1.20)$ & 0.405 \\
\hline $\begin{array}{l}\text { Hemoglobin } \\
(\mathrm{g} / \mathrm{dL})\end{array}$ & 13.0]11.5-14.6] & $13.0[11.0-14.8]$ & $13.0[11.7-14.6]$ & $0.95(0.86-1.06)$ & $0.99(0.89-1.10)$ & 0.820 \\
\hline Urea $(\mathrm{mg} / \mathrm{dL})$ & $40.0[28.0-65.0]$ & $47.5[33.0-95.0]$ & $38.0[26.7-55.2]$ & $1.02(1.01-1.02)$ & $1.01(1.01-1.02)$ & $<0.001$ \\
\hline $\begin{array}{c}\text { Creatinine } \\
(\mathrm{mg} / \mathrm{dL})\end{array}$ & $1.1[0.9-1.5]$ & $1.3[1.0-2.0]$ & $1.1[0.9-1.4]$ & $1.54(1.21-1.96)$ & $1.47(1.17-1.85)$ & 0.001 \\
\hline BUN/Creatinine & $15.9[12.1-20.5]$ & $16.8[12.7-23.9]$ & $15.7[12.0-20.0]$ & $1.06(1.03-1.10)$ & $1.05(1.01-1.08)$ & 0.004 \\
\hline $\begin{array}{l}\text { Sodium } \\
(\mathrm{mmol} / \mathrm{L})\end{array}$ & $\begin{array}{l}135.1 \\
\quad[131.6-138.6]\end{array}$ & $\begin{array}{l}134.9 \\
\quad[131.1-138.8]\end{array}$ & $\begin{array}{l}135.1 \\
{[131.7-138.4]}\end{array}$ & $1.02(0.98-1.06)$ & $1.02(0.98-1.06)$ & 0.315 \\
\hline $\begin{array}{l}\text { Potassium } \\
(\mathrm{mmol} / \mathrm{L})\end{array}$ & $4.5[4.1-4.8]$ & $4.5[4.0-4.9]$ & $4.4[4.1-4.8]$ & $1.19(0.81-1.74)$ & $1.06(0.71-1.58)$ & 0.770 \\
\hline $\begin{array}{l}\text { Blood sugar } \\
(\mathrm{mg} / \mathrm{dL})\end{array}$ & $\begin{array}{l}172.0 \\
{[118.0-244.0]}\end{array}$ & $\begin{array}{l}203.5 \\
\quad[124.0-273.5]\end{array}$ & $\begin{array}{l}162.0 \\
{[114.0-233.5]}\end{array}$ & $1.00(1.00-1.01)$ & $1.00(1.00-1.01)$ & 0.094 \\
\hline $\mathrm{FBS}(\mathrm{mg} / \mathrm{dL})$ & $\begin{array}{l}193.0 \\
{[141.5-259.0]}\end{array}$ & $\begin{array}{l}190.5 \\
{[142.0-244.0]}\end{array}$ & $\begin{array}{l}202.0 \\
{[140.0-272.0]}\end{array}$ & $1.00(0.99-1.00)$ & $1.00(0.99-1.00)$ & 0.436 \\
\hline $\mathrm{CRP}(\mathrm{mg} / \mathrm{L})$ & $68.1[31.2-103.6]$ & $90.6[50.1-135.8]$ & $61.0[26.6-95.8]$ & $1.01(1.00-1.01)$ & $1.01(1.00-1.01)$ & $<0.001$ \\
\hline $\mathrm{ESR}(\mathrm{mm} / \mathrm{h})$ & $61.5[37.0-90.0]$ & $60.0[36.0-90.0]$ & $64.0[38.0-89.0]$ & $1.00(0.99-1.01)$ & $1.00(0.99-1.01)$ & 0.739 \\
\hline $\begin{array}{l}\text { hs-cTnI } \\
\quad(\mathrm{pg} / \mathrm{mL})\end{array}$ & $6.0[1.8-16.1]$ & $11.1[4.2-69.6]$ & $4.8[1.5-11.9]$ & $1.00(1.00-1.01)$ & $1.00(1.00-1.01)$ & 0.011 \\
\hline AST (U/L) & $52.0[37.0-68.0]$ & $61.0[42.0-75.5]$ & $47.0[36.0-61.0]$ & $1.01(1.00-1.02)$ & $1.01(1.00-1.02)$ & 0.013 \\
\hline ALT (U/L) & $37.0[27.0-52.0]$ & $40.0[28.5-52.5]$ & $36.0[25.0-51.7]$ & $1.01(1.00-1.01)$ & $1.00(1.00-1.01)$ & 0.376 \\
\hline ALP (U/L) & $\begin{array}{l}176.5 \\
{[140.2-231.0]}\end{array}$ & $\begin{array}{l}179.0 \\
{[147.0-264.0]}\end{array}$ & $\begin{array}{l}176.0 \\
{[138.0-227.0]}\end{array}$ & 1.00 & 1.00 & 0.494 \\
\hline \multicolumn{7}{|c|}{ Preadmission drug use In-hospital outcomes } \\
\hline $\begin{array}{l}\text { Hospital length } \\
\text { of stay (day) }\end{array}$ & $6.0[3.0-9.5]$ & $7.0[3.0-12.0]$ & $6.0[3.0-9.0]$ & $1.04(1.01-1.07)$ & $1.04(1.01-1.07)$ & 0.016 \\
\hline Severity & $304(77.4 \%)$ & $111(92.5 \%)$ & $193(70.7 \%)$ & $5.11(2.47-10.58)$ & $4.78(2.28-10.05)$ & $<0.001$ \\
\hline ICU admission & $76(19.3 \%)$ & $60(50.0 \%)$ & $16(5.9 \%)$ & $16.06(8.65-29.83)$ & $\begin{array}{l}21.95 \\
\quad(10.94-44.02)\end{array}$ & $<0.001$ \\
\hline $\begin{array}{l}\text { Multi-organ } \\
\text { damage }\end{array}$ & $103(26.2 \%)$ & $66(55.0 \%)$ & $37(13.6 \%)$ & $7.80(4.73-12.85)$ & $7.92(4.68-13.39)$ & $<0.001$ \\
\hline
\end{tabular}

ACEIs angiotensin-converting-enzyme inhibitors, $A L P$ alkaline phosphatase, $A L T$ alanine aminotransferase, $A S T$ aspartate aminotransferase, $A R B s$ angiotensin II receptor blockers, $B M I$ body mass index, $B U N$ blood urea nitrogen, $C R P$ C-reactive protein, DPP-4 inhibitors dipeptidyl peptidase-4 inhibitors, $E S R$ erythrocyte sedimentation rate, $F B S$ fasting blood sugar, $h s$ - $c T n I$ high-sensitivity cardiac troponin I, $R B C$ red blood cells, SII systemic immune-inflammation index, WBC white blood cells

*Data are presented as mean \pm standard deviation, number (\%), or median [interquartile range]

${ }^{\dagger}$ Statistically significant P-values are bolded

in patients with DM and COVID-19, ACEIs/ARBs, and statins are commonly used by patients with DM; [13]. Moreover, the beneficial, neutral, or hazardous effects of antiplatelets are controversial [14-16]. A meta-analysis on 8990 patients with COVID-19 concluded hazard of mortality and severe disease was reduced by $30 \%$ with statin use [HR (95\% CI): 0.70 (0.53-0.94), P-value: 0.01] [45]. Another meta-analysis on 3449 patients with COVID19 revealed statins did not have any impact on mortality [OR (95\% CI): $0.78(0.50-1.21)$, P-value: 0.26$]$ or severity [OR(95\% CI): 1.64 (0.51-5.23), P-value: 0.41) [46]. Another retrospective, cohort, and theoretical studies emphasized on beneficial effects of statins in patients with COVID-19 [47-49].

SARS-CoV-2-induced renin-angiotensin aldosterone system (RAAS) imbalance, comprised of reduced ACE2 expression (and a subsequent elevated Ang-2 activity), plays a role in severe COVID-19 complications. Because SARS-CoV-2 uses ACE2 for cell entry, it has been thought that ACEIs/ARBs can have potential effects on 
Table 3 Logistic regression analysis of preadmission drug use for prediction of severity, MAE, and 7-month all-cause mortality

\begin{tabular}{|c|c|c|c|c|c|c|c|c|c|}
\hline \multirow{2}{*}{$\begin{array}{l}\text { Preadmiss- } \\
\text { sion drug use }\end{array}$} & \multicolumn{3}{|c|}{ Severity } & \multicolumn{3}{|c|}{ MAE } & \multicolumn{3}{|c|}{ 7-month all-cause mortality } \\
\hline & OR & $95 \% \mathrm{CI}$ & $\mathrm{P}^{\dagger}$ & OR & $95 \%$ CI & $\mathrm{P}^{\dagger}$ & HR & $95 \% \mathrm{CI}$ & $\mathrm{P}^{\dagger}$ \\
\hline \multicolumn{10}{|l|}{ Metformin } \\
\hline Model 1 & 1.29 & $0.79-2.12$ & 0.303 & 1.35 & $0.81-2.24$ & 0.244 & 0.88 & $0.60-1.24$ & 0.489 \\
\hline Model 2 & 1.24 & $0.67-2.28$ & 0.491 & 1.35 & $0.71-2.60$ & 0.361 & 1.13 & $0.73-1.74$ & 0.575 \\
\hline \multicolumn{10}{|l|}{ Sulfonylureas } \\
\hline Model 1 & 1.83 & $0.91-3.71$ & 0.092 & 1.86 & $0.90-3.87$ & 0.094 & 1.09 & $0.69-1.74$ & 0.702 \\
\hline Model 2 & 2.05 & $0.87-4.79$ & 0.099 & 2.12 & $0.85-5.29$ & 0.108 & 1.23 & $0.75-2.02$ & 0.410 \\
\hline \multicolumn{10}{|c|}{ DPP-4 inhibitors } \\
\hline Model 1 & 1.48 & $0.47-4.63$ & 0.501 & 1.34 & $0.43-4.21$ & 0.616 & 0.97 & $0.39-2.41$ & 0.948 \\
\hline Model 2 & 3.20 & $0.64-15.99$ & 0.156 & 3.13 & $0.61-16.02$ & 0.171 & 1.16 & $0.41-3.27$ & 0.773 \\
\hline \multicolumn{10}{|l|}{ Insulin } \\
\hline Model 1 & 0.67 & $0.33-1.33$ & 0.253 & 0.77 & $0.37-1.58$ & 0.475 & 0.87 & $0.51-1.48$ & 0.606 \\
\hline Model 2 & 1.07 & $0.44-2.71$ & 0.842 & 1.44 & $0.52-3.94$ & 0.482 & 0.91 & $0.50-1.65$ & 0.757 \\
\hline \multicolumn{10}{|l|}{ ACEIs/ARBs } \\
\hline Model 1 & 1.62 & $0.87-3.01$ & 0.128 & 1.83 & $0.96-3.48$ & 0.066 & 1.09 & $0.70-1.71$ & 0.696 \\
\hline Model 2 & 1.64 & $0.77-3.52$ & 0.203 & 1.77 & $0.78-3.99$ & 0.169 & 1.13 & $0.67-1.90$ & 0.655 \\
\hline \multicolumn{10}{|l|}{ Beta blockers } \\
\hline Model 1 & 1.25 & $0.63-2.47$ & 0.525 & 1.19 & $0.59-2.40$ & 0.624 & 0.80 & $0.48-1.32$ & 0.378 \\
\hline Model 2 & 1.06 & $0.46-2.44$ & 0.897 & 0.99 & $0.41-2.40$ & 0.988 & 0.78 & $0.43-1.39$ & 0.391 \\
\hline \multicolumn{10}{|c|}{ Calcium channel blockers } \\
\hline Model 1 & 2.34 & $0.78-7.01$ & 0.130 & 2.13 & $0.71-6.42$ & 0.177 & 1.25 & $0.67-2.33$ & 0.472 \\
\hline Model 2 & 4.26 & $0.93-19.63$ & 0.063 & 3.25 & $0.70-15.10$ & 0.132 & 1.80 & $0.94-3.44$ & 0.074 \\
\hline \multicolumn{10}{|l|}{ Statins } \\
\hline Model 1 & 1.57 & $0.86-2.86$ & 0.139 & 1.65 & $0.89-3.08$ & 0.114 & 0.73 & $0.46-1.16$ & 0.188 \\
\hline Model 2 & 1.40 & $0.69-2.86$ & 0.354 & 1.46 & $0.68-3.13$ & 0.336 & 0.86 & $0.52-1.40$ & 0.534 \\
\hline \multicolumn{10}{|l|}{ Antiplatelets } \\
\hline Model 1 & 1.53 & $0.81-2.89$ & 0.190 & 1.70 & $0.87-3.31$ & 0.121 & 0.80 & $0.51-1.25$ & 0.331 \\
\hline Model 2 & 1.41 & $0.64-3.11$ & 0.391 & 1.54 & $0.65-3.67$ & 0.325 & 1.09 & $0.67-1.76$ & 0.734 \\
\hline
\end{tabular}

ACEIs angiotensin-converting enzyme inhibitors, ARBs angiotensin II receptor blockers, AST aspartate aminotransferase, $C R P$ C-reactive protein, DPP-4 inhibitors dipeptidyl peptidase-4 inhibitors, MAE major adverse event, $W B C$ white blood cells

Model 1: Multivariate regression adjusted for age, sex, and comorbidities (hypertension, cardiac disease, cerebrovascular disease, chronic lung disease, malignancy); (Hosmer and Lemeshow $\mathrm{P} \geq 0.318$ )

Model 2: Multivariate regression adjusted for age, sex, comorbidities (hypertension, cardiac disease, cerebrovascular disease, chronic lung disease, malignancy), WBC, CRP, urea, and AST; (Hosmer and Lemeshow $\mathrm{P} \geq 0.131$ )

†Statistically significant P-values are bolded patients with COVID-19 but several systematic reviews and meta-analyses done on the effects of ACEIs/ARBs on patients with COVID-19 confirmed these medications are safe [50]. A meta-analysis showed the risk of COVID-19 infection reduced by $26 \%$ in 330,780 patients using ACEIs compared with non-users [pooled OR (95\% CI): $0.74(0.65-0.85)$, P-value $<0.001$ ], but no effect was observed in 275,621 patients using ARBs compared with nonusers [pooled OR (95\% CI): 0.90 (0.79-1.02), P-value: 0.11]. Another meta-analysis on 169 hospitals in Asia, Europe, and North America revealed neutral effects of ACEIs/ARBs on SARS-COV-2 infection, hospital or ICU admission, severe or critical illness, or related death in the total population, but decreased severe or critical illness and death in Asians [OR(95\%): $0.37(0.16-0.89), \mathrm{I}^{2}=83 \%$ and OR(95\%): $0.62(0.39-0.99), \mathrm{I}^{2}=0 \%$ respectively), increased ICU admission in North Americans [OR(95\% CI): 1.75 (1.37-2.23), $\left.\mathrm{I}^{2}=0 \%\right]$ and increased death in Europeans [OR(95\% CI): $\left.1.68(1.05-2.70), \mathrm{I}^{2}=82 \%\right](51)$.

\section{Limitations}

Our study has some limitations. First, the impact of inhospital and long-term glycemic control on outcomes was not evaluated in this study. Second, it was an observational 
study; no causal inference can be made, and the relationship should be interpreted as an association, and randomized trials are needed in the future. Third, it was a single-center study, and multicenter studies are needed to judge.

\section{Conclusions}

The potential clinical characteristics associated with longterm mortality could guide clinicians to identify patients with poor prognoses. Preadmission use of sulfonylureas and ACEIs/ARBs were associated with borderline increased risk of in-hospital adverse outcomes.

Supplementary Information The online version contains supplementary material available at https://doi.org/10.1007/s40200-021-00901-4.

Acknowledgements We acknowledge all healthcare workers involved in the diagnosis and treatment of patients in Sina Hospital. We are indebted to the Research Development Center of Sina Hospital for its support.

Author contributions MP: concept and design, drafting of the manuscript, revision of the manuscript. FC: data Interpretation, statistical analysis, drafting of the manuscript, revision of the manuscript. A.Hadadi: concept and design, data acquisition, supervision. SK: concept and design, data acquisition, supervision. MM: concept and design, data acquisition, supervision. SK: concept and design, data interpretation, statistical analysis, drafting of the manuscript, revision of the manuscript. AA: data interpretation, statistical analysis, drafting of the manuscript, revision of the manuscript. ME: concept and design, data acquisition, supervision. HA: concept and design, data interpretation, statistical analysis, drafting of the manuscript, supervision. MMH: concept and design, data acquisition, supervision. MRK: concept and design, data acquisition, supervision. RSM: concept and design, data acquisition, supervision. SHS: concept and design, data acquisition, supervision. SKS: concept and design, data acquisition, supervision. HR: concept and design, data acquisition, supervision. NG: concept and design, data acquisition. PH: data Interpretation, statistical analysis, drafting of the manuscript, revision of the manuscript.

Funding Tehran University of Medical Sciences supported this study. The funding source had no role in the study design, data collection, data analysis, data interpretation, writing of the manuscript, or submission decision.

Data availability Data are available upon a reasonable request to the corresponding author.

\section{Declarations}

Conflict of interest All authors declare that they have no competing interests.

Ethical approval This study was approved by the Ethics Committee of Tehran University of Medical Sciences (IR.TUMS.VCR. REC.1399.018).

\section{References}

1. Zhu N, Zhang D, Wang W, Li X, Yang B, Song J, et al. A Novel Coronavirus from Patients with Pneumonia in China, 2019. N Engl J Med. 2020;382(8):727-33.

2. Cucinotta D, Vanelli M. WHO declares COVID-19 a pandemic. Acta Biomed. 2020;91(1):157-60.

3. Shabto JM, Loerinc L, O'Keefe GA, O'Keefe J. Characteristics and outcomes of COVID-19 positive patients with diabetes managed as outpatients. Diabetes Res Clin Pract. 2020;164:108229.

4. Grasselli G, Zangrillo A, Zanella A, Antonelli M, Cabrini L, Castelli A, et al. Baseline characteristics and outcomes of 1591 patients infected with SARS-CoV-2 admitted to ICUs of the Lombardy Region, Italy. JAMA. 2020;323(16):1574-81.

5. Lukito AA, Pranata R, Henrina J, Lim MA, Lawrensia S, Suastika $\mathrm{K}$. The effect of metformin consumption on mortality in hospitalized COVID-19 patients: a systematic review and meta-analysis. Diabetes Metab Syndr. 2020;14(6):2177-83.

6. Cariou B, Hadjadj S, Wargny M, Pichelin M, Al-Salameh A, Allix I, et al. Phenotypic characteristics and prognosis of inpatients with COVID-19 and diabetes: the CORONADO study. Diabetologia. 2020;63(8):1500-15.

7. Wargny M, Potier L, Gourdy P, Pichelin M, Amadou C, Benhamou PY, et al. Predictors of hospital discharge and mortality in patients with diabetes and COVID-19: updated results from the nationwide CORONADO study. Diabetologia. 2021;64(4):778-94.

8. Rhee SY, Lee J, Nam H, Kyoung D-S, Kim DJ. Effects of a DPP-4 inhibitor and RAS blockade on clinical outcomes of patients with diabetes and COVID-19. Diabetes Metab J. 2021;45(2):251-9.

9. Solerte SB, D'Addio F, Trevisan R, Lovati E, Rossi A, Pastore I, et al. Sitagliptin treatment at the time of hospitalization was associated with reduced mortality in patients with type 2 diabetes and COVID-19: a multicenter, case-control, retrospective, observational study. Diabetes Care. 2020;43(12):2999-3006.

10. Kim MK, Jeon J-H, Kim S-W, Moon JS, Cho NH, Han E, et al. The clinical characteristics and outcomes of patients with moderate-to-severe coronavirus disease 2019 infection and diabetes in Daegu, South Korea. Diabetes Metab J. 2020;44(4):602.

11. Izzi-Engbeaya C, Distaso W, Amin A, Yang W, Idowu O, Kenkre JS, et al. Adverse outcomes in COVID-19 and diabetes: a retrospective cohort study from three London teaching hospitals. BMJ Open Diabetes Res Care. 2021;9(1):e001858.

12. Dalan R, Ang LW, Tan WYT, Fong SW, Tay WC, Chan YH, et al. The association of hypertension and diabetes pharmacotherapy with COVID-19 severity and immune signatures: an observational study. Eur Heart J Cardiovasc Pharmacother. 2021;7(3):e48-51.

13. Sparks MA, South A, Welling P, Luther JM, Cohen J, Byrd JB, et al. Sound science before quick judgement regarding RAS blockade in COVID-19. Clin J Am Soc Nephrol. 2020;15(5):714-6.

14. Yan F, Huang F, Xu J, Yang P, Qin Y, Lv J, et al. Antihypertensive drugs are associated with reduced fatal outcomes and improved clinical characteristics in elderly COVID-19 patients. Cell Discov. 2020;6(1):77.

15. Pinto-Sietsma SJ, Flossdorf M, Buchholz VR, Offerhaus J, Bleijendaal H, Beudel M, et al. Antihypertensive drugs in COVID-19 infection. Eur Heart J Cardiovasc Pharmacother. 2020;6(6):415-6.

16. Banik J, Mezera V, Köhler C, Schmidtmann M. Antiplatelet therapy in patients with Covid-19: a retrospective observational study. Thrombosis Update. 2020;2:100026.

17. Soleimani A, Kazemian S, Karbalai Saleh S, Aminorroaya A, Shajari Z, Hadadi A, et al. Effects of angiotensin receptor blockers (ARBs) on in-hospital outcomes of patients with hypertension and confirmed or clinically suspected COVID-19. Am J Hypertens. 2020;33(12):1102-11. 
18. Karbalai Saleh S, Oraii A, Soleimani A, Hadadi A, Shajari Z, Montazeri M, et al. The association between cardiac injury and outcomes in hospitalized patients with COVID-19. Intern Emerg Med. 2020;15(8):1415-24.

19. Pazoki M, Keykhaei M, Kafan S, Montazeri M, Mirabdolhagh Hazaveh M, Sotoodehnia M, et al. Risk indicators associated with in-hospital mortality and severity in patients with diabetes mellitus and confirmed or clinically suspected COVID-19. J Diabetes Metab Disord. 2021;20(1):1-11.

20. World HealthOrganization. Clinical management of severe acute respiratory infection when novel coronavirus $(2019-\mathrm{nCoV})$ infection is suspected: interim guidance, 28 January 2020. World Health Organization; 2020.

21. Daily Situation Report on Coronavirus disease (COVID-19) in Iran; March 14, 2020. Arch Acad Emerg Med. 2020;8(1):e24.

22. Iran's Diagnostic Therapeutic Flowchart for COVID-19; 1 th -7 th editions. http://treatment.sbmu.ac.ir/index.jsp?pageid=63989\&p=1.

23. Classification and Diagnosis of Diabetes. Standards of medical care in diabetes-2020. Diabetes Care. 2020;43(Suppl 1):S14-s31.

24. Thygesen K, Mair J, Katus H, Plebani M, Venge P, Collinson P, et al. Recommendations for the use of cardiac troponin measurement in acute cardiac care. Eur Heart J. 2010;31(18):2197-204.

25. Ranieri VM, Rubenfeld GD, Thompson BT, Ferguson ND, Caldwell E, Fan E, et al. Acute respiratory distress syndrome: the Berlin Definition. JAMA. 2012;307(23):2526-33.

26. Khwaja A. KDIGO clinical practice guidelines for acute kidney injury. Nephron Clin Pract. 2012;120(4):c179-184.

27. Dufour DR, Lott JA, Nolte FS, Gretch DR, Koff RS, Seeff LB. Diagnosis and monitoring of hepatic injury. II. Recommendations for use of laboratory tests in screening, diagnosis, and monitoring. Clin Chem. 2000;46(12):2050-68.

28. Saha S, Al-Rifai RH, Saha S. Diabetes prevalence and mortality in COVID-19 patients: a systematic review, meta-analysis, and meta-regression. J Diabetes Metab Disord. 2021;20(1):1-12.

29. Zhou F, Yu T, Du R, Fan G, Liu Y, Liu Z, et al. Clinical course and risk factors for mortality of adult inpatients with COVID19 in Wuhan, China: a retrospective cohort study. Lancet. 2020;395(10229):1054-62.

30. Li B, Yang J, Zhao F, Zhi L, Wang X, Liu L, et al. prevalence and impact of cardiovascular metabolic diseases on COVID-19 in China. Clin Res Cardiol. 2020;109(5):531-8.

31. Lippi G, Wong J, Henry BM. Hypertension in patients with coronavirus disease 2019 (COVID-19): a pooled analysis. Pol Arch Intern Med. 2020;130(4):304-9.

32. Henry BM, Lippi G. Chronic kidney disease is associated with severe coronavirus disease 2019 (COVID-19) infection. Int Urol Nephrol. 2020;52(6):1193-4.

33. Hansrivijit $\mathrm{P}$, Qian C, Boonpheng B, Thongprayoon C, Vallabhajosyula $\mathrm{S}$, Cheungpasitporn $\mathrm{W}$, et al. Incidence of acute kidney injury and its association with mortality in patients with COVID19: a meta-analysis. J Investig Med. 2020;68(7):1261-70.

34. Yuan SY, Breslin JW, Perrin R, Gaudreault N, Guo M, Kargozaran $\mathrm{H}$, et al. Microvascular permeability in diabetes and insulin resistance. Microcirculation. 2007;14(4-5):363-73.

35. Xiao L, Sakagami H, Miwa N. ACE2: the key molecule for understanding the pathophysiology of severe and critical conditions of COVID-19: demon or angel? Viruses. 2020;12(5):491.

36. Azoulay L, Suissa S. Sulfonylureas and the risks of cardiovascular events and death: a methodological meta-regression analysis of the observational studies. Diabetes Care. 2017;40(5):706-14.

37. Varvaki Rados D, Catani Pinto L, Reck Remonti L, Bauermann Leitão C, Gross JL. The association between sulfonylurea use and all-cause and cardiovascular mortality: a meta-analysis with trial sequential analysis of randomized clinical Trials. PLoS Med. 2016;13(4):e1001992.
38. Pasquel FJ, Fayfman M, Umpierrez GE. Debate on insulin vs noninsulin use in the hospital setting-is it time to revise the guidelines for the management of inpatient diabetes? Curr Diab Rep. 2019;19(9):65.

39. Apicella M, Campopiano MC, Mantuano M, Mazoni L, Coppelli A, Del Prato S. COVID-19 in people with diabetes: understanding the reasons for worse outcomes. Lancet Diabetes Endocrinol. 2020;8(9):782-92.

40. Bramante CT, Ingraham NE, Murray TA, Marmor S, Hovertsen $\mathrm{S}$, Gronski J, et al. Observational study of metformin and risk of mortality in patients hospitalized with covid-19. medRxiv. 2020. https://doi.org/10.1101/2020.06.19.20135095.

41. Pérez-Belmonte LM, Torres-Peña JD, López-Carmona MD, Ayala-Gutiérrez MM, Fuentes-Jiménez F, Huerta LJ, et al. Mortality and other adverse outcomes in patients with type 2 diabetes mellitus admitted for COVID-19 in association with glucose-lowering drugs: a nationwide cohort study. BMC Med. 2020;18(1):359.

42. Gao Y, Liu T, Zhong W, Liu R, Zhou H, Huang W, et al. Risk of metformin in patients with type 2 diabetes With COVID-19: a preliminary retrospective report. Clin Transl Sci. 2020;13(6):1055-9.

43. Agarwal S, Schechter C, Southern W, Crandall JP, Tomer Y. Preadmission diabetes-specific risk factors for mortality in hospitalized patients with diabetes and coronavirus disease 2019. Diabetes Care. 2020;43(10):2339-44.

44. Crouse AB, Grimes T, Li P, Might M, Ovalle F, Shalev A. Metformin use is associated with reduced mortality in a diverse population with COVID-19 and diabetes. Front Endocrinol. 2021;11:1081.

45. Kow CS, Hasan SS. Meta-analysis of effect of statins in patients with COVID-19. Am J Cardiol. 2020;134:153-5.

46. Hariyanto TI, Kurniawan A. Statin therapy did not improve the in-hospital outcome of coronavirus disease 2019 (COVID-19) infection. Diabetes Metab Syndr. 2020;14(6):1613-5.

47. Zhang X-J, Qin J-J, Cheng X, Shen L, Zhao Y-C, Yuan Y, et al. In-hospital use of statins is associated with a reduced risk of mortality among individuals with COVID-19. Cell Metab. 2020;32(2):176-187.e4.

48. De Spiegeleer A, Bronselaer A, Teo JT, Byttebier G, De Tré G, Belmans L, et al. The effects of ARBs, ACEis, and statins on clinical outcomes of COVID-19 infection among nursing home residents. J Am Med Dir Assoc. 2020;21(7):909-914.e2.

49. Sanchis-Gomar F, Perez-Quilis C, Favaloro EJ, Lippi G. Statins and other drugs: Facing COVID-19 as a vascular disease. Pharmacol Res. 2020;159:105033.

50. Hoevenaar M, Goossens D, Roorda J. Angiotensin-converting enzyme 2, the complement system, the Kallikrein-Kinin system, type-2 diabetes, interleukin-6, and their interactions regarding the complex COVID-19 pathophysiological crossroads. J Renin Angiotensin Aldosterone Syst. 2020;21(4):1470320320979097.

51. Patoulias D, Katsimardou A, Stavropoulos K, Imprialos K, Kalogirou MS, Doumas M. Renin-angiotensin system inhibitors and COVID-19: a systematic review and meta-analysis. Evidence for significant geographical disparities. Curr Hypertens Rep. 2020;22(11):90.

Publisher's Note Springer Nature remains neutral with regard to jurisdictional claims in published maps and institutional affiliations. 